\title{
Description of the derived categories of tubular algebras in terms of dimension vectors
}

\author{
Hongbo Lv, Zhongmei Wang \\ School of Mathematical Science, University of Jinan, Jinan 250022, P.R.China \\ School of Control Science and Engineering, Shandong University, Jinan 250061, P.R.China \\ Email: lvhongbo356@163.com; wangzhongmei211@sohu.com
}

\begin{abstract}
In this paper, we give a description of the derived category of a tubular algebra by calculating the dimension vectors of the objects in it.
\end{abstract}

Keywords:derived categories; tubular algebra; dimension vectors

\section{Introduction}

Let $\Lambda$ be a basic connected algebra over an algebraically closed field $k$. We denote by $\bmod \Lambda$ the category of all finitely generated right $\Lambda$-modules and by ind $\Lambda$ a full subcategory of $\bmod \Lambda$ containing exactly one representative of each isomorphism class of indecomposable $\boldsymbol{\Lambda}$-modules. For a $\boldsymbol{\Lambda}$-module $\boldsymbol{M}$, we denote the dimension vector by $\operatorname{dim} \boldsymbol{M}$. The bounded derived category of mod $\Lambda$ is denoted by $\boldsymbol{D}^{t}(\boldsymbol{\Lambda})$. We denote the Grothendieck group of $\Lambda$ by $\boldsymbol{K}_{0}(\Lambda)$, AuslanderReiten translation by $\tau$, the Cartan matrix by $C_{\Lambda}$. Let $\hat{\Lambda}$ be the repetitive algebra of $\Lambda$, mod $\hat{\Lambda}$ the stable module category. When the global dimension of $\Lambda$ is finite, $C_{\Lambda}$ is invertible by [1], and $\boldsymbol{D}^{b}(\boldsymbol{\Lambda})$ is equivalent to $\bmod \hat{\boldsymbol{\Lambda}}$ as triangulated categories by [2].

By [1], a tubular extension $A$ of a tame-concealed algebra

of extension type $\mathrm{T}=(2,2,2,2),(3,3,3),(4,4,2)$ or $(6,3$, 2)

is called a tubular algebra. For example, the canonical tubular algebras of $\mathrm{T}(2,2,2,2)$ is determined by the following quiver with relations.

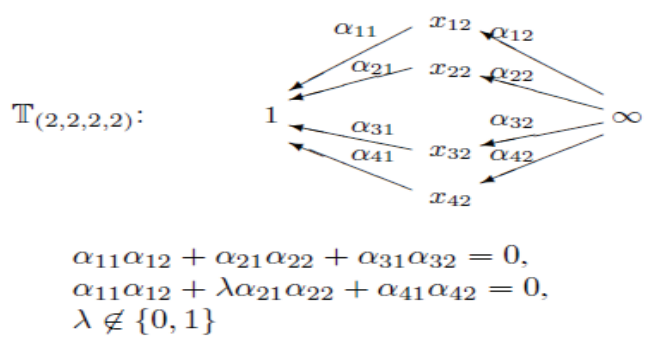

By [1], global dimension of a tubular algebra $A$ is 2 , then $\boldsymbol{D}^{b}(A)$ is equivalent to mod $\hat{A}$. And tubular algebras of the same extension type are tilt-cotilt equivalent, see [3]. Then we only consider the derived categories of canonical tubular algebras, whose structures are given in [4].

$$
D^{b}(A)=\underset{r \in Q}{\vee} T_{r}
$$

where (1) for any $r \in Q, T_{r}$ is the standard stable $\mathrm{P}_{1}(k)$ tubular family of type $\mathrm{T}$;

(2) for any $r \in Q, T_{r}$ is separating $\underset{s<r}{\vee} T_{s}$ from $\underset{r<u}{\vee} T_{u}$.

Based on the results above, we give a description of the derived category of a canonical tubular algebra by calculating the dimension vectors of the objects in it.

\section{Description of The Derived Categories of Tubular Algebras In Terms Of Dimension Vectors}

In this section, let $A$ be a canonical tubular algebra of type $\mathrm{T}$.

Definition 1.1. ([1]) Let $n$ be the rank of Grothendieck group $K_{0}(A), C_{A}$ the Cartan matrix of $A$. Then

(1) The Coxeter matrix $\Phi_{A}$ is defined by $-C_{A}{ }^{-T} C_{A}$;

(2) The quadratic form $\chi_{A}$ in $\mathbb{Z}_{n}$ is defined by

$$
\chi_{A}(\alpha)=\frac{1}{2} \alpha\left(-C_{A}^{-T}+C_{A}^{-1}\right) \alpha^{T}
$$

for any $\alpha$ in $\mathbb{Z}_{n}$.

(3) Let $h_{0}, h_{\infty}$ be the positive generators of $\operatorname{rad} \chi_{A}$. For an $A$-module $M$, define

where

$$
\operatorname{index}(M)=-\frac{l_{0}(\underline{\operatorname{dim}} M)}{l_{\infty}(\underline{\operatorname{dim}} M)}
$$

$$
\begin{array}{r}
l_{0}\left(\underline{\operatorname{dim} M)}=h_{0} C_{A}^{-T}\left(\underline{\operatorname{dim} M)^{T},}\right.\right. \\
l_{\infty}\left(\underline{\operatorname{dim} M)=h_{\infty} C_{A}^{-T}(\underline{\operatorname{dim}} M)^{T} .}\right. \text { particular, } \\
\text { In }{ }_{\alpha \in \operatorname{rad}} \chi_{A}, \alpha=r_{0} h_{0}+r_{\infty} h_{\infty}, \\
r_{0}, r_{\infty} \in \mathbb{Z} . \text { Then, index }(\alpha)=\frac{r_{\infty}}{r_{0}} .
\end{array}
$$$$
\text { In particular, for any }
$$ 
(4) $\alpha \in \operatorname{rad} \chi_{A}$ is called a real (respectively, imaginary) root,

if $\chi_{A}(\alpha)=\frac{1}{2} \alpha\left(-C_{A}{ }^{-T}+C_{A}{ }^{-1}\right) \alpha^{T}=1$ (respectively, $=0$ ).

It is well known that there exists a "minimal " imaginary root $\delta$ such that $\operatorname{rad} \chi_{A}=\mathbb{Z} \delta$.

Now we recall some results in [4]. Let $\Lambda$ be a finite dimensional $k$-algebra and $\widehat{\Lambda}$ the repetitive algebra. Denote

by $P(\widehat{\Lambda})$ the subgroup of $K_{0}(\widehat{\Lambda})$ generated by the dimension vectors of indecomposable projective $\widehat{\Lambda}-$ modules.

Lemma 1.2. $K_{0}(\widehat{\Lambda})=K_{0}(\Lambda)+P(\widehat{\Lambda})$.

Definition 1.3. Let $\pi_{\Lambda}: K_{0}(\widehat{\Lambda}) \rightarrow K_{0}(\Lambda)$ be the projective morphism. Define $\underline{\operatorname{dim}}^{\Lambda}: \bmod \widehat{\Lambda} \rightarrow K_{0}(\Lambda)$ where for any $\widehat{\Lambda}$-module $X, \underline{\operatorname{dim}}^{\Lambda} X=\pi_{\Lambda}(\underline{\operatorname{dim}} X)$.

Lemma 1.4. Let $\Phi_{\Lambda}$ be the Coxeter matrix of $\Lambda, \hat{\tau}$ the Auslander-Reiten translation of $\widehat{\Lambda}$. Then

$$
\underline{\operatorname{dim}}^{\Lambda} \hat{\tau} X=(\underline{\operatorname{dim}} X) \Phi_{\Lambda} .
$$

Note that if $\Lambda$ has finite global dimension, we have a triangulated equivalence: $\eta: D^{b}(\Lambda) \rightarrow \underline{\bmod \widehat{\Lambda}}$. For an object $X^{\cdot} \in D^{b}(\Lambda)$, define $\underline{\operatorname{dim}} X^{\cdot}=\sum_{i}(-1)^{i} \underline{\operatorname{dim}} X^{i}$.

Then we have

Lemma 1.5. $\underline{\operatorname{dim}} X^{\cdot}=\underline{\operatorname{dim}}^{\Lambda} \eta\left(X^{\bullet}\right)$. [5]

By representation theory of Auslander-Reiten quivers in and the results above, we have a method to describing the derived category of a canonical tubular algebra in terms of dimension vectors.

Theorem 1.6. Let $A$ be a canonical tubular algebra of type

$\mathrm{T}$, the rank of $K_{0}(A)$ be $n$. Then

(1) Let $\hat{\delta}$ be the minimal imaginary root in $K_{0}(\widehat{A})$ corresponding the $\mathrm{P}_{1}(k)$ - tubular family $T_{r}$, and let $\delta=\underline{\operatorname{dim}}^{A}(\hat{\delta})$. Then $\delta$ is determined by $\chi_{A}(\delta)=0$.

(2) Let $X$ be an object in the bottom of a tube of rank $r$ in $T_{r}$. Then $\underline{\operatorname{dim}}^{A} X$ is determined by the following:

(*) $\left\{\chi_{A}\left(\underline{\operatorname{dim}}^{A} X\right)=\frac{1}{2} \underline{\operatorname{dim}}^{A} X\left(-C_{A}{ }^{-T}+C_{A}{ }^{-1}\right)\left(\underline{\operatorname{dim}}^{A} X\right)^{T}=1\right.$ $\underline{\operatorname{dim}}^{A} X+\left(\underline{\operatorname{dim}}^{A} X\right) \Phi_{A}+\cdots+\left(\underline{\operatorname{dim}}^{A} X\right) \Phi_{A}{ }^{r-1}=\delta$.
Proof. (1) By [4], $\delta=r_{0} h_{0}+r_{\infty} h_{\infty} \in \operatorname{rad} \chi_{A}$, and thus $\chi_{A}(\delta)=0 . \quad$ Since $\quad \operatorname{index}(\delta)=\frac{r_{\infty}}{r_{0}} \in \mathbb{Q}, \quad$ where $r_{0}, r_{\infty} \in \mathbb{Z}$, and $\left(r_{0}, r_{\infty}\right)=1$, it suffices to calculating $r_{0}$ and $r_{\infty}$.

(2) Directly from Lemma 1.4 and 1.5 .

Example 1.7. Now let $A$ be a canonical tubular algebra of type $\mathrm{T}(2,2,2,2)$. The Cartan matrix and Coxeter matrix are as following:

$C_{A}=\left(\begin{array}{llllll}1 & 1 & 1 & 1 & 1 & 2 \\ 0 & 1 & 0 & 0 & 0 & 1 \\ 0 & 0 & 1 & 0 & 0 & 1 \\ 0 & 0 & 0 & 1 & 0 & 1 \\ 0 & 0 & 0 & 0 & 1 & 1 \\ 0 & 0 & 0 & 0 & 0 & 1\end{array}\right), \Phi_{A}=\left(\begin{array}{cccccc}-1 & -1 & -1 & -1 & -1 & -2 \\ 0 & 1 & 0 & 0 & 0 & -1 \\ 0 & 0 & 1 & 0 & 0 & -1 \\ 0 & 0 & 0 & 1 & 0 & -1 \\ 0 & 0 & 0 & 0 & 1 & -1 \\ 0 & 0 & 0 & 0 & 0 & 1\end{array}\right)$

For each object $\quad X \in \underline{\bmod } \widehat{A}, \quad$ denote

$$
\underline{\operatorname{dim}}^{A} X=\left(x_{1}, x_{2}, \cdots, x_{6}\right) \text {, }
$$

Then $\chi_{A}\left(\underline{\operatorname{dim}}^{A} X\right)=\sum_{i=2}^{5}\left(x_{i}-\frac{x_{1}+x_{6}}{2}\right)^{2}$.

(1) Description of the minimal imaginary root $\delta$.

Case 1. If $r_{0}+r_{\infty} \equiv 1(\bmod 2)$

$\delta_{1}=\left(2 r_{0}, r_{0}+r_{\infty}, r_{0}+r_{\infty}, r_{0}+r_{\infty}, r_{0}+r_{\infty}, 2 r_{\infty}\right)$.

Case 2. If $r_{0}+r_{\infty} \equiv 0(\bmod 2)$

$\delta_{2}=\left(r_{0}, \frac{r_{0}+r_{\infty}}{2}, \frac{r_{0}+r_{\infty}}{2}, \frac{r_{0}+r_{\infty}}{2}, \frac{r_{0}+r_{\infty}}{2}, r_{\infty}\right)$.

(2) Description of $\underline{\operatorname{dim}}^{A} X$ where $X$ is an object in the bottom of a tube of rank 2 .

If $\delta=\delta_{1}$, that is $r_{0}+r_{\infty} \equiv 1(\bmod 2),(*)$ in Theorem 1.6 should be as follows:

$$
\left\{\begin{array}{c}
\sum_{i=1}^{6} x_{i}^{2}-\sum_{i=2}^{5} x_{1} x_{i}-\sum_{i=2}^{5} x_{6} x_{i}+2 x_{1} x_{6}=1 \\
\sum_{i=2}^{5} x_{i}-2 x_{6}=2 r_{0} \\
\sum_{i=2}^{5} x_{i}-x_{1}-x_{6}=r_{0}+r_{\infty} \\
\sum_{i=2}^{5} x_{i}-2 x_{1}=2 r_{\infty}
\end{array}\right.
$$


$\underline{\mathrm{d} \mathrm{m}}^{A} X=\left(\dot{\eta_{0}}, \frac{r_{0}+r_{\infty}-1}{2}, \frac{r_{0}+r_{\infty}-1}{2}\right.$,

$$
\left.\frac{r_{0}+r_{\infty}+1}{2}, \frac{r_{0}+r_{\infty}+1}{2}, r_{\infty}\right)
$$

$\underline{\mathrm{d} \mathrm{m}}^{A} \hat{\tau} X=\left(1 r_{0}, \frac{r_{0}+r_{\infty}+1}{2}, \frac{r_{0}+r_{\infty}+1}{2}\right.$,

$$
\left.\frac{r_{0}+r_{\infty}-1}{2}, \frac{r_{0}+r_{\infty}-1}{2}, r_{\infty}\right)
$$

(ii)

$$
\begin{aligned}
\underline{\operatorname{dim}}^{A} X= & \left(r_{0}, \frac{r_{0}+r_{\infty}-1}{2}, \frac{r_{0}+r_{\infty}+1}{2},\right. \\
& \left.\frac{r_{0}+r_{\infty}-1}{2}, \frac{r_{0}+r_{\infty}+1}{2}, r_{\infty}\right)
\end{aligned}
$$

$\underline{\operatorname{dim}}^{A} \hat{\tau} X=\left(r_{0}, \frac{r_{0}+r_{\infty}+1}{2}, \frac{r_{0}+r_{\infty}-1}{2}\right.$,

$$
\left.\frac{r_{0}+r_{\infty}+1}{2}, \frac{r_{0}+r_{\infty}-1}{2}, r_{\infty}\right)
$$

(iii)

$\underline{\operatorname{dim}}^{A} X=\left(r_{0}, \frac{r_{0}+r_{\infty}-1}{2}, \frac{r_{0}+r_{\infty}+1}{2}\right.$,

$$
\left.\frac{r_{0}+r_{\infty}+1}{2}, \frac{r_{0}+r_{\infty}-1}{2}, r_{\infty}\right)
$$

$\underline{\operatorname{dim}}^{A} \hat{\tau} X=\left(r_{0}, \frac{r_{0}+r_{\infty}+1}{2}, \frac{r_{0}+r_{\infty}-1}{2}\right.$,

$$
\left.\frac{r_{0}+r_{\infty}-1}{2}, \frac{r_{0}+r_{\infty}+1}{2}, r_{\infty}\right)
$$

(iv)

$$
\begin{aligned}
\underline{\mathrm{d} \mathrm{m}}^{A} X= & \left(\dot{r}_{\mathrm{b}}+1, \frac{r_{0}+r_{\infty}+1}{2}, \frac{r_{0}+r_{\infty}+1}{2},\right. \\
& \left.\frac{r_{0}+r_{\infty}+1}{2}, \frac{r_{0}+r_{\infty}+1}{2}, r_{\infty}+1\right) \\
\underline{\mathrm{d} \mathrm{m}}^{A} \hat{\tau} X= & \left(1 r_{0}-1, \frac{r_{0}+r_{\infty}-1}{2}, \frac{r_{0}+r_{\infty}-1}{2},\right. \\
& \left.\frac{r_{0}+r_{\infty}-1}{2}, \frac{r_{0}+r_{\infty}-1}{2}, r_{\infty}-1\right)
\end{aligned}
$$

If $\delta=\delta_{2},(*)$ in Theorem 1.6 should be as follows:

$$
\begin{aligned}
& \left(\sum_{i=1}^{6} x_{i}^{2}-\sum_{i=2}^{5} x_{1} x_{i}-\sum_{i=2}^{5} x_{6} x_{i}+2 x_{1} x_{6}=1\right. \\
& \sum_{i=2}^{5} x_{i}-2 x_{6}=r_{0} \\
& \sum_{i=2}^{5} x_{i}-x_{1}-x_{6}=\frac{r_{0}+r_{\infty}}{2} \\
& \sum_{i=2}^{5} x_{i}-2 x_{1}=r_{\infty} \\
& \text { Then, } \sum_{i=2}^{5}\left(x_{i}-\frac{r_{0}+r_{\infty}{ }^{2}}{4}\right)^{2}=1 \text {. }
\end{aligned}
$$

case 2. When $r_{0}+r_{l} \equiv 2(\bmod 4)$, we have four different tubes of rank 2 .

(i)

$$
\begin{aligned}
\underline{\operatorname{dim}}^{A} X= & \left(\frac{r_{0}-1}{2}, \frac{r_{0}+r_{\infty}+2}{4}, \frac{r_{0}+r_{\infty}-2}{4},\right. \\
& \left.\frac{r_{0}+r_{\infty}-2}{4}, \frac{r_{0}+r_{\infty}-2}{4}, \frac{r_{\infty}-1}{2}\right)
\end{aligned}
$$

$\underline{\operatorname{dim}}^{A} \hat{\tau} X=\left(\frac{r_{0}+1}{2}, \frac{r_{0}+r_{\infty}-2}{4}, \frac{r_{0}+r_{\infty}+2}{4}\right.$,

$$
\left.\frac{r_{0}+r_{\infty}+2}{4}, \frac{r_{0}+r_{\infty}+2}{4}, \frac{r_{\infty}+1}{2}\right)
$$

(ii)

$$
\begin{aligned}
\underline{\operatorname{dim}}^{A} X= & \left(\frac{r_{0}-1}{2}, \frac{r_{0}+r_{\infty}-2}{4}, \frac{r_{0}+r_{\infty}+2}{4},\right. \\
& \left.\frac{r_{0}+r_{\infty}-2}{4}, \frac{r_{0}+r_{\infty}-2}{4}, \frac{r_{\infty}-1}{2}\right)
\end{aligned}
$$

$\underline{\operatorname{dim}}^{A} \hat{\tau} X=\left(\frac{r_{0}+1}{2}, \frac{r_{0}+r_{\infty}+2}{4}, \frac{r_{0}+r_{\infty}-2}{4}\right.$,

$$
\left.\frac{r_{0}+r_{\infty}+2}{4}, \frac{r_{0}+r_{\infty}+2}{4}, \frac{r_{\infty}+1}{2}\right)
$$

(iii)

$$
\begin{aligned}
\underline{\mathrm{d} \mathrm{m}}^{A} X= & \left(\frac{r_{0}-1}{2}, \frac{r_{0}+r_{\infty}-2}{4}, \frac{r_{0}+r_{\infty}-2}{4},\right. \\
& \left.\frac{r_{0}+r_{\infty}+2}{4}, \frac{r_{0}+r_{\infty}-2}{4}, \frac{r_{\infty}-1}{2}\right)
\end{aligned}
$$

$$
\begin{aligned}
\mathrm{d} \mathrm{m}^{A} \hat{\tau} X= & \left(\frac{r_{0}+1}{2}, \frac{r_{0}+r_{\infty}+2}{4}, \frac{r_{0}+r_{\infty}+2}{4},\right. \\
& \left.\frac{r_{0}+r_{\infty}-2}{4}, \frac{r_{0}+r_{\infty}+2}{4}, \frac{r_{\infty}+1}{2}\right)
\end{aligned}
$$

(iv) 


$$
\begin{aligned}
\underline{\mathrm{d} \mathrm{m}}^{A} X= & \left(\frac{r_{q}-1}{2}, \frac{r_{0}+r_{\infty}-2}{4}, \frac{r_{0}+r_{\infty}-2}{4},\right. \\
& \left.\frac{r_{0}+r_{\infty}-2}{4}, \frac{r_{0}+r_{\infty}+2}{4}, \frac{r_{\infty}-1}{2}\right) \\
\frac{\mathrm{d} \mathrm{m}}{} \hat{\tau} X= & \left(\frac{r_{0}+1}{2}, \frac{r_{0}+r_{\infty}+2}{4}, \frac{r_{0}+r_{\infty}+2}{4},\right. \\
& \left.\frac{r_{0}+r_{\infty}+2}{4}, \frac{r_{0}+r_{\infty}-2}{4}, \frac{r_{\infty}+1}{2}\right)
\end{aligned}
$$

case 3. When $r_{0}+r_{l} \equiv 0(\bmod 4)$, we have four different tubes of rank 2 .

(i)

$$
\begin{aligned}
& \underline{\mathrm{d} \mathrm{m}}^{A} X \dot{\mp}\left(\frac{r_{0}+1}{2}, \frac{r_{0}+r_{\infty}}{4}, \frac{r_{0}+r_{\infty}}{4}\right. \text {, } \\
& \left.\frac{r_{0}+r_{\infty}}{4}, \frac{r_{0}+r_{\infty}+4}{4}, \frac{r_{\infty}+1}{2}\right) \\
& \underline{\mathrm{d} \mathrm{m}}^{A} \hat{\tau} X \mathrm{i}=\left(\frac{r_{0}-1}{2}, \frac{r_{0}+r_{\infty}}{4}, \frac{r_{0}+r_{\infty}}{4},\right. \\
& \left.\frac{r_{0}+r_{\infty}}{4}, \frac{r_{0}+r_{\infty}-4}{4}, \frac{r_{\infty}-1}{2}\right)
\end{aligned}
$$

(ii)

$$
\begin{aligned}
& \underline{\mathrm{d} \mathrm{m}}^{A} X \dot{\mathrm{m}}\left(\frac{r_{0}+1}{2}, \frac{r_{0}+r_{\infty}}{4}, \frac{r_{0}+r_{\infty}}{4},\right. \\
&\left.\frac{r_{0}+r_{\infty}+4}{4}, \frac{r_{0}+r_{\infty}}{4}, \frac{r_{\infty}+1}{2}\right) \\
& \frac{\mathrm{d} \mathrm{m}}{} \hat{\tau} X \mathrm{i}=\left(\frac{r_{0}-1}{2}, \frac{r_{0}+r_{\infty}}{4}, \frac{r_{0}+r_{\infty}}{4},\right. \\
&\left.\frac{r_{0}+r_{\infty}-4}{4}, \frac{r_{0}+r_{\infty}}{4}, \frac{r_{\infty}-1}{2}\right)
\end{aligned}
$$

(iii)

$$
\begin{aligned}
\underline{\operatorname{dim}}^{A} X= & \left(\frac{r_{0}+1}{2}, \frac{r_{0}+r_{\infty}}{4}, \frac{r_{0}+r_{\infty}+4}{4},\right. \\
& \left.\frac{r_{0}+r_{\infty}}{4}, \frac{r_{0}+r_{\infty}}{4}, \frac{r_{\infty}+1}{2}\right) \\
\underline{\operatorname{dim}}^{A} \hat{\tau} X= & \left(\frac{r_{0}-1}{2}, \frac{r_{0}+r_{\infty}}{4}, \frac{r_{0}+r_{\infty}-4}{4},\right. \\
& \left.\frac{r_{0}+r_{\infty}}{4}, \frac{r_{0}+r_{\infty}}{4}, \frac{r_{\infty}-1}{2}\right)
\end{aligned}
$$

(iv)

$$
\begin{aligned}
\underline{\operatorname{dim}}^{A} X= & \left(\frac{r_{0}+1}{2}, \frac{r_{0}+r_{\infty}+4}{4}, \frac{r_{0}+r_{\infty}}{4},\right. \\
& \left.\frac{r_{0}+r_{\infty}}{4}, \frac{r_{0}+r_{\infty}}{4}, \frac{r_{\infty}+1}{2}\right)
\end{aligned}
$$

$$
\begin{aligned}
\underline{\operatorname{dim}}^{A} \hat{\tau} X= & \left(\frac{r_{0}-1}{2}, \frac{r_{0}+r_{\infty}-4}{4}, \frac{r_{0}+r_{\infty}}{4},\right. \\
& \left.\frac{r_{0}+r_{\infty}}{4}, \frac{r_{0}+r_{\infty}}{4}, \frac{r_{\infty}-1}{2}\right)
\end{aligned}
$$

\section{Acknowledgment}

The authors would like to thank the referee for his or her valuable suggestions and comments. The first-named author thanks NSF of China (Grant No. 11126300) and of Shandong Province (Grant No. ZR2011AL015) for support.

\section{REFERENCES}

[1] C.M.Ringel, Tame algebras and integral quadratic forms, Lecture Notes in Math. 1099. Springer Verlag, 1984.

[2] D.Happel, Triangulated categories in the representation theory of finite dimensional algebras, Lecture Notes series 119. Cambridge Univ. Press, 1988.

[3] D.Happel, On the derived category of a finitedimensional algebra. Comment. Math. Helv. 62(1987), 339_389.

[4] D.Happel, C.M.Ringel, The derived category of a tubular

algebra. LNM1273, Berlin-Heidelbelrg-NewYork: Springer-Verlag, 1986:156_180.

[5] W.Crawley-Boevey, Lectures on Representations of Quivers. Preprint. 